\title{
The Relationship Between Vitamin D Serum Levels and Rheumatoid Arthritis Activity in a Sample of Syrian Patients- a cross-sectional observational study
}

Zaynab Alourfi ( $\nabla$ zaynabarfi@hotmail.com )

Damascus University

Nisreen Aldahhan

Damascus University

Research Article

Keywords: Rheumatoid Arthritis Activity, Vitamin D, Syria

Posted Date: March 22nd, 2021

DOI: https://doi.org/10.21203/rs.3.rs-276142/v1

License: (c) (1) This work is licensed under a Creative Commons Attribution 4.0 International License. Read Full License 


\section{Abstract}

Background. Rheumatoid Arthritis (RA) is a systemic auto immune disease that mainly affects joints. It might cause functional disability and increased morbidity and mortality.

Vitamin D was reported to have an immunomodulation role in reducing the inflammation process in many auto immune diseases; RA is one of them.

The aim was to determine the relationship between serum vitamin D levels and rheumatoid arthritis activity in a sample of Syrian patients.

Methods This cross-sectional observational study was carried out in Al-Mouwasat and Al-Assad University Hospitals(Damascus University - Syria). RA patients were diagnosed according to the American College of Rheumatology (ACR) and the European League Against Rheumatism(EULAR)classification criteria for rheumatoid arthritis 2010 standards.

Results Vitamin D was significantly higher in RA patients in remission $(27.92 \pm 13 \mathrm{ng} / \mathrm{ml})$ than in patients with active RA $(21.22 \pm 13 \mathrm{ng} / \mathrm{ml})$. There was an inverse relationship between serum vitamin $D$ levels and RA activity.

Conclusion Serum vitamin D levels were inversely correlated with RA activity in a sample of Syrian RA patients.

\section{Background}

Rheumatoid Arthritis (RA) is a systemic auto immune disease affecting $1 \%$ of adults of all ages. However, it is seen more frequently during the fifth and sixth age decade in females and males respectively and is more common in women (male to female ratio of 1:3). [1-3]. It mainly affects the synovial membrane, causing inflammation and proliferation, loss of articular cartilage and periarticular erosions although other organs may be affected. The disease may progress to functional disability with long, frequent sickness absence leading to disability pension. Eberhardt, et al.(2007) reported over a period of 15 years of follow up, that work disability in RA patients increased from $28 \%$ to $44 \%$.. Additionally, the return of RA patients to work is usually very difficult [3].

However, early diagnosis and treatment were shown to give good recovery outcomes. RA has been found to be triggered by genetic, epigenetic (gene polymorphisms in several cytokine genes, altered miRNA, and altered DNA methylation..etc) and environmental (smoking, air pollutants, diet, obesity and infections) factors. [4]

Vitamin D levels were reported to be lower in patients with autoimmune diseases than in healthy controls. [5-7]. Several animal studies have shown the role of vitamin D supplementation in controlling many autoimmune diseases such as arthritis and autoimmune encephalomyelitis. However, in humans, studies had conflicting results [8-9]. Other studies compared vitamin D intake to the prevalence of autoimmune 
diseases such as RA and type 1 diabetes, and inflammatory conditions such as cardiovascular disease and atherosclerosis $[10,11]$.

Vitamin D deficiency is known to be highly prevalent in Syria [12]. This study aims to estimate serum vitamin D levels in a sample of confirmed RA Syrian patients and determine its relationship with RA activity.

\section{Methods}

This cross-sectional observational study was conducted at Al-Mouwasat University Hospital and AlAssad University Hospital in Damascus, between March 15, 2017 and December 31, 2019. Both are central, and teaching hospitals that serve as major tertiary referral hospitals for Damascus and other cities in Syria. (http://auhd.edu.sy/en).

\section{Patients}

RA patients were diagnosed according to the American College of Rheumatology (ACR) and the European LeagueAgainst Rheumatism(EULAR)classification criteria for rheumatoid arthritis 2010 standards.

\section{Exclusion criteria}

Patients with mixed connective tissue disease, or any conditions that affect vitamin D levels, or vitamin D metabolism (hepatic or renal insufficiency), as well as patients with malabsorption, or those taking drugs that affect vitamin D metabolism such as phenytoin, phenobarbital, carbamazepine, isoniazid, rifampicin, and theophylline, and PTH, were excluded from this study.

Patients on steroids were included due to the controversial effects of steroids on levels of Vitamin D. [13].Patients who took vitamin $D$ were included in this study as this cross-sectional research aims to determine serum levels of vitamin $D$ and disease activity in real time, regardless of vitamin $D$ source.

\section{Main outcome variable}

RA activity was determined using Disease Activity score 28 ESR (DAS28-ESR). Patients were categorized according to their activity score into remission (score $<2.6$ ), mild (between 2.6 and 3.2), moderate (between 3.2 and 5.1), and severe activity (> 5.1)

Patients were divided into two main groups: Group 1 (remission), and Group 2 (mild, moderate or severe disease activity).

\section{Study factors}


250HD levels were measured using automated electrochemiluminescence immunoassay (Elecsys 2010 analyzers, Roche Diagnostics GmbH, Mannheim, Germany). Seventy-five nmol/L (30 ng/mL) or above was the cut off point for vitamin D normal levels according to Endocrine Society cut-off values [14].

\section{Procedures}

This study included RA patients attending outpatients clinics at Al- Mouwasat and Al-Assad University Hospitals or admitted to the hospital and examined by the same rheumatologist.

Fasting blood sample was obtained from each participant. Blood samples were analyzed immediately by the same team of laboratory technicians and the same method was used throughout the study period, using kits provided by the same manufacturer [12].

Study protocol was approved by the Damascus University institutional review board. A written informed consent was obtained from each participant.

\section{Statistical analysis}

Statistical analysis was carried out using Predictive Analytics Software Statistics (PASW Statistics) version 18. Chi-square test was used to analyze group differences for categorical variables and differences were assessed using Mann-Whitney $U$ test. Results were presented as mean and standard deviation (SD). Pearson correlation test was used to estimate the relationship between vitamin $D$ and RA activity. A p value less than 0.05 was considered significant.

\section{Results}

100 RA patient were included, $29 \%$ were males, and $71 \%$ were females. Age ranged between 22 and 85 years, with an average of $51 \pm 13$ years.

Vitamin D serum level mean value was $22.96 \pm 13.27 \mathrm{ng} / \mathrm{ml}$, (between 3 and $63 \mathrm{ng} / \mathrm{ml}$ ).

Active disease was observed in $74 \%$ of patients whereas $26 \%$ had total remission Mild, moderate and severe disease activity was recorded in $25 \%, 23 \%$, and $26 \%$ of patients respectively.

Using the Mann-Whitney $U$ test, vitamin D was significantly lower in the active RA group than the remission group (Table 1). $(P=0.02)$. There was an inverse negative relationship between vitamin $D$ and RA activity groups $(P=0.005)$ (Fig 1$)$. 
Table 1

Vitamin D in RA remission and active groups

\begin{tabular}{|llll|}
\hline RA Activity & $\mathbf{n}$ & $\begin{array}{l}\text { Vitamin D ng/ml } \\
\text { Mean } \pm \text { Std }\end{array}$ & $\mathbf{p}$ \\
\hline Remission & 26 & $28 \pm 13$ & 0.02 \\
\hline Active & 74 & $21 \pm 13$ & \\
\hline RA: Rheumatoid arthritis, N: number of patients \\
\hline
\end{tabular}

\section{Discussion}

Although a plethora of research has been published on the relationship between vitamin D and RA activity, results are conflicting [7]. This might be explained by the fact that apart from the differences in assay methodology, circulating $250 \mathrm{HD}$ level is affected by environmental (sun exposure, latitude, season) and biological factors (age, gender, race/ethnicity, skin color, body mass index), as well as socioeconomic status, medication use, and vitamin D dietary intake (from fortified food or supplements).

This cross-sectional study was conducted in Damascus which is located at latitude $33.25^{\circ}$ north (moderate latitudes) and longitude $36.31^{\circ}$ east, between March 15, 2017 and December 31, 2019 which may eliminate serum $250 H D$ seasonal variations.

A vitamin D level less than $30 \mathrm{ng} / \mathrm{ml}$ (either insufficient or deficient) was recorded in $70 \%$ of our RA patients. This suggested that vitamin D levels were low in most RA patients which is consistent with published research (Gheita, Cecchetti, Mateen, and Khoja). In contrast, no differences in vitamin D levels between healthy and RA people were reported in several other studies (5).

We found that Vitamin D was inversely correlated to RA activity, which was similar to results obtained by other researchers [15-16]. However, Herly et al, Cooles et al, Matsumoto [7] and Packchotanon et al [17] found no relationship between vitamin D and RA activity.

A meta-analysis carried out by Lin et al reported that "Stronger inverse relationship between RA disease activity and serum 25-OHD3 in studies from developing countries, and in low-latitude climates". This may be similar to the situation observed in this study of Syrian patients. Moreover, most of the reported studies were observational or cross-sectional by design, that only report the association between RA disease and vitamin D.

Many systematic reviews were conducted on the relationship between vitamin $\mathrm{D}$ and rheumatoid disease, but they did not ultimately reach conclusive results $[7,18,19]$.

The main limitation of this study is the small sample size. It is therefore difficult to generalize our results on all the Syrian population. However, to the best of our knowledge, this is the first study that highlights this issue in Syria. 
In conclusion, there was an inverse relationship between serum vitamin D levels and RA disease activity in Syrian RA patients included in this study. Other studies with larger sample size and interventional design are needed.

\section{Abbreviations}

RA: Rheumatoid Arthritis, ACR: American College of Rheumatology, EULAR: the European LeagueAgainst Rheumatism. DAS28-ESR:Disease Activity score 28 ESR, SD: Standard Deviation,

\section{Declarations}

\section{Ethics approval and consent to participate:}

The study protocol was approved by the Damascus University institutional review board. It was designed and conducted in accordance with the declaration of Helsinki and the principles of Good Clinical Practice.

A written informed consent was obtained from each participant.

\section{Consent for publication:}

Not applicable

Availability of data and materials:

The datasets used and analysed during the current study are available from the authors upon reasonable request and with permission of Damascus University.

\section{Competing interests:}

None

\section{Funding:}

None

\section{Authors' contributions:}

NA designed the study and underwent a patients clinical assessment. ZA was responsible for data analysis. Both authors contributed to interpretation of the results, preparation and review of the manuscript, and approval of the final manuscript for publication.

\section{Acknowledgements:}

The authors are grateful to Prof. Salwa Al Sheikh and Prof Majed Abboud for general support, and to Dr. B. Boufana for manuscript English language editing. 


\section{References}

1-Gary S Firestein, The disease formerly known as rheumatoid arthritis, Arthritis Res Ther. 2014; 16(3): 114.

2-Eberhardt K;LarssonBM;NivedK;et al: Work disability in Rheumatoid arthritis development over 15 years and evaluation of predictive factors over time. J Rheumatol 2007,34:481-487.

3- Hansen SM, Hetland ML, Pedersen J, Østergaard M, Rubak TS, Bjorner JB Work ability in rheumatoid arthritis patients: a register study on the prospective risk of exclusion and probability of returning to work. Rheumatology (Oxford). 2017 Jul 1;56(7):1135-1143. doi: 10.1093/rheumatology/kex064. PMID: 28371921

4- Choy E; Understanding the dynamics: pathways involved in the pathogenesis of Rheumatoid arthritis . Rheumatology 2012:3-11.

5-Sassi F; TamoneC ;D’Amelio P. Vitamin D: Nutrient, Hormone, and Immunomodulator. Nutrients 2018.

6-Giustina et al, Controversies in Vitamin D: A Statement From the Third International Conference, JBMR Plus 2020 Nov 10;4(12):e10417

7-Stephanie R.Harrison; Danyang L; Louisa E;et al: Vitamin D, Autoimmune Disease and Rheumatoid Arthritis.Springer2020:

8 -Gunasekar, P.; Swier, V.J.; Fleegel, J.P.;et al. Vitamin D and macrophage polarization in epicardial adipose tissue of atherosclerotic swine. PLoS ONE 2018, 13.

9 - Lin J, Liu J, Davies ML, Chen W (2016) Serum vitamin D level and rheumatoid arthritis disease activity: review and meta-analysis. PLoS ONE 11:e0146351

10 - Song, G.G.; Bae, S.-C.; Lee, Y.H. Association between vitamin D intake and the risk of rheumatoid arthritis:A meta-analysis. Clin. Rheumatol. 2012, 31, 1733-1739.

11- Dong, J.-Y.; Zhang, W.-G.; Chen, J.J.; Zhang, Z.-L.;et al . Vitamin D intake and risk of type 1 diabetes: A meta-analysis of observational studies. Nutrients 2013, 5, 3551-3562

12 - R. Sayed-Hassan, N. Abazid, Z. Alourfi, Relationship between25-hydroxyvitamin D concentrations, serum calcium, and parathyroidhormone in apparently healthy Syrian people, Arch. Osteoporos. 9 (2014) 176.

13 - RobienK,OppeneerS,Kelly J, et al. Drug vitamin D interactions: A systematic Review of the Literature. NutrClin Pract.2013

14 - Zaynab Alourfi., et al. "Rethinking Vitamin D Deficiency Cut-Off Point: A Study Among Healthy Syrian Adults". EC Endocrinology and Metabolic Research 4.3 (2019): 72-82. 
15 - Yang, L $\square$ Hongyan, W. Impact of vitamin D deficiency on clinical parameters in treatment naïve rheumatoid arthritis patients.ZeitschriftfürRheumatologie .2018

16 - Narendra M, Sumit Pal Singh C, Ravinder G, Anil 1, SarabjotK.Assessment of Vitamin D in Rheumatid Arthritis and Its Correlation with Disease Activity.Journal of Natural Science, Biology and Medicine.2018

17 - Baker JF, Baker DG, Toedter G, et al. Associations between vitamin D, disease activity, and clinical response to therapy in rheumatoid arthritis. ClinExpRheumatol2012; 30:

18 - Pakchotanon R, Chaiamnuay S, Narongroeknawin P, et al. The association between serum vitamin D level and disease activity in Thai rheumatoid arthritis patients. International Journal of Rheumatic Diseases 2016;19:

19 - Bragazzi.N.L, Watad.A . Neumann.S,et al. Vitamin D and rheumatoid arthritis: an ongoing mystery. Curr Opin Rheumatol 2017 Jul;29(4):378-388.

\section{Figures}




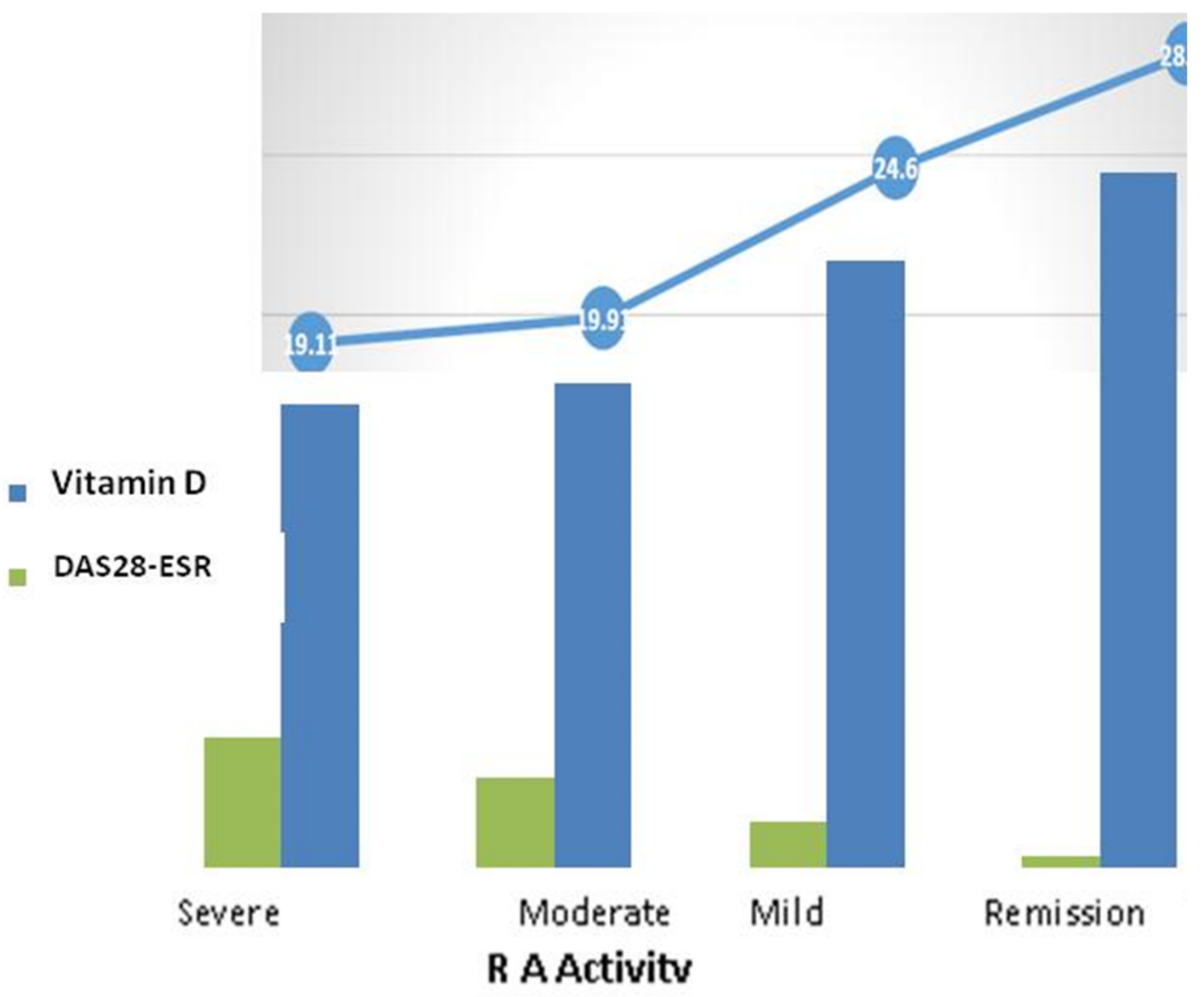

Figure 1

The relationship between vitamin D value and activity level DAS28-ESR:Disease Activity score 28 ESR 